\title{
Diagnosis of Plasmodium vivax by Loop-Mediated Isothermal Amplification in Febrile Patient Samples from Loreto, Perú
}

\author{
Oscar Nolasco, ${ }^{1 \star}$ Beronica Infante,${ }^{2}$ Juan Contreras-Mancilla, ${ }^{1}$ Sandra Incardona, ${ }^{3}$ Xavier C. Ding, ${ }^{3}$ Dionicia Gamboa, ${ }^{1,2,4}$ and \\ Katherine Torres ${ }^{1,2}$ \\ ${ }^{1}$ Instituto de Medicina Tropical "Alexander von Humboldt" Universidad Peruana Cayetano Heredia, Lima, Perú; ${ }^{2}$ Laboratorios de Investigación y \\ Desarrollo, Facultad de Ciencias y Filosofía, Universidad Peruana Cayetano Heredia, Lima, Perú; ${ }^{3}$ Foundation for Innovative New Diagnostics, \\ Geneva, Switzerland; ${ }^{4}$ Departamento de Ciencias Celulares y Moleculares, Facultad de Ciencias y Filosofía, Universidad Peruana Cayetano \\ Heredia, Lima, Perú
}

\begin{abstract}
Plasmodium vivax is co-endemic with Plasmodium falciparum in Peru, and optimum management requires distinguishing these two species in the blood of patients. For the differential identification of $P$. vivax and other Plasmodium spp., the Loopamp ${ }^{\text {TM }}$ Malaria Pan Detection Kit in combination with the Loopamp Malaria Pv Detection Kit (Eiken Chemical Co. Ltd., Tokyo, Japan) was used to evaluate 559 whole blood samples collected in 2017 from febrile patients with suspected malaria attending different health facilities in the Loreto region. The Loopamp Malaria Pan Detection Kit showed a sensitivity of $87.7 \%$ (95\% Cl: 83.5-91.9) and a specificity of $94.4 \%$ (95\% Cl: 91.9-96.9) and good agreement with PCR (Cohen's kappa 0.8266, 95\% Cl: 0.7792-0.874). By comparison, the Loopamp Malaria Pv Detection Kit showed a similar sensitivity $(84.4 \%, 95 \% \mathrm{Cl}$ : 79.0-89.7) and specificity $(92.4 \%, 95 \% \mathrm{Cl}$ : 89.7-95.0) and substantial agreement with PCR (Cohen's kappa: 0.7661, 95\% Cl: 0.7088-0.8234).
\end{abstract}

In the Peruvian Amazonia, good sensitivity and specificity for diagnosis of Plasmodium infection are essential for efficient malaria control. In this region, there are two species of Plasmodium with different prevalence; the reported incidence of Plasmodium vivax infectious is nearly four times higher than Plasmodium falciparum infections. ${ }^{1}$ The detection and identification are a challenge because of the high prevalence of submicroscopic and asymptomatic infections confirmed by epidemiological studies. ${ }^{2,3}$ Specific detection of $P$. vivax is required so that a radical cure combining chloroquine and primaquine to eliminate both blood- and liver-stage parasites can be administered. ${ }^{4}$

Microscopy including thin and thick blood smear is the most commonly used diagnostic method for detection and identification of Plasmodium and as such remains a standard of practice. Although microscopy can achieve an excellent detection limit when overseen by an expert (5-20 parasites per microliter of blood $[\mathrm{p} / \mu \mathrm{L}])$, this method is often associated with misdiagnosis at points of care (POC) when performed by a health worker with limited proficiency and in infections with low parasitemia $(10-100 \mathrm{p} / \mu \mathrm{L})$. $^{5,6}$ Rapid diagnostic tests (RDTs) represent an alternative for malaria diagnosis by offering an easy-to-use and quick method. Their sensitivity for $P$. falciparum detection is approximately $100 \mathrm{p} / \mu \mathrm{L}$; however, current RDTs for $P$. vivax are usually slightly less sensitive, translating to a significant proportion of false negatives because of the low parasitemia typically associated with infections by this species. ${ }^{7-9}$ Molecular diagnostic methods can achieve a sensitivity as low as $0.02 \mathrm{p} / \mu^{10}$ and are understandably the best option to detect and identify all Plasmodium infections, including those in asymptomatic patients. ${ }^{11}$ However, only isothermal amplification techniques such as the loop-mediated isothermal amplification (LAMP) can be effectively applied in areas with limited laboratory capacity

\footnotetext{
*Address correspondence to Oscar Nolasco, Instituto de Medicina Tropical "Alexander von Humboldt" Universidad Peruana Cayetano Heredia, Av. Honorio Delgado 430 SMP, Lima 15102, Perú. E-mail: oscar.nolasco.c@upch.pe
}

because DNA amplification occurs at a constant temperature and the amplification product can be visually detected by turbidity or fluorescence, without any additional sample manipulation. $^{12}$

The commercially available Loopamp Pan/Pf Malaria Kit has been previously used in multiple settings, including in the Loreto region in Peruvian Amazonia, showing a high operational capacity in the field and a high sensitivity. ${ }^{13}$ Herein, we report the clinical evaluation of a $P$. vivax infection by using the Loopamp ${ }^{\mathrm{TM}}$ Malaria Pv Detection Kit (Human, ref. 975000), which complements the already available LAMP products designed for detection of pan Plasmodium spp. and $P$. falciparum.

The Loopamp ${ }^{\mathrm{TM}}$ Malaria Pan Detection Kit (Human, ref. 977000 ) and the $P$. vivax commercial assay were evaluated in a retrospective laboratory study against 559 frozen ethylenediaminetetraacetic acid-anticoagulated venous whole blood samples. Samples were collected from febrile patients consecutively attending four different health facilities located in the communities of San Juan, Lupuna, Santa Clara, and Quistococha in the Loreto region from March to June 2017. The study was approved by the Institutional Ethical Committee of Universidad Peruana Cayetano Heredia (code 100068).

All the samples were evaluated by two independent microscopy readings and by molecular diagnostic methods in two reference laboratories. A real-time PCR diagnosis with a species-specific melting probe and unidirectional sequencing by using the noncoding region between the cytb and cox1 genes was performed at Labor Limbach Laboratory (Heidelberg, Germany) for species determination. ${ }^{14}$ A second analysis by nested PCR and bidirectional sequencing for species determination were performed at Microsynth laboratory (Balgach, Switzerland), using the cytb gene of the Plasmodium mitochondrial DNA. ${ }^{15}$

The LAMP kits were used according to the manufacturer's instructions. ${ }^{16}$ In brief, nucleic acid was extracted using the boil and spin method, where $60 \mu \mathrm{L}$ of whole blood sample was dispensed into a 1.5-mL tube containing $60 \mu \mathrm{L}$ of DNA extraction buffer $(400 \mathrm{mM} \mathrm{NaCl}, 40 \mathrm{mM}$ Tris $\mathrm{pH} 6.5 \%$ and $0.4 \%$ SDS), mixed for 5 minutes at $95^{\circ} \mathrm{C}$ in a heat block, and 
TABLE 1

Performance evaluation of microscopy and Loopamp Malaria Pan Detection Kit for Plasmodium detection, using PCR as the reference method

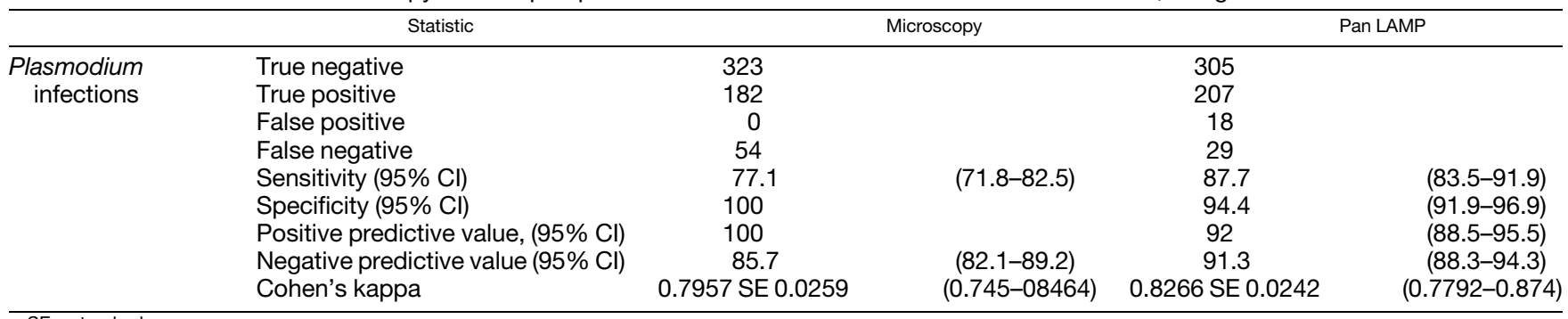

SE $=$ standard error.

centrifuged at $10,000 \mathrm{~g}$ for 3 minutes. The supernatant was recovered, and $30 \mu \mathrm{L}$ was transferred into a tube containing $345 \mu \mathrm{L}$ of sterile water. The extracted and diluted DNA $(30 \mu \mathrm{L})$ was added to the Pan LAMP and PV LAMP reaction tubes, with resuspension of the reagents according to the manufacturer's instructions. Samples were incubated for 40 minutes at $65^{\circ} \mathrm{C}$ in a heat block, followed by 5 minutes at $80^{\circ} \mathrm{C}$ to stop the reaction. DNA amplification was detected based on fluorescence observed in the reaction mix when exposed to an ultraviolet lamp. Each LAMP reaction was judged three times, and the final report was the result of two or three equal visual observations.

Of the 559 samples, 225 (40.6\%) showed positive reaction to Plasmodium by the Loopamp Malaria Pan Detection Kit, whereas 180 (32.2\%) samples showed positive reaction to $P$. vivax by the Loopamp Malaria Pv Detection Kit. PCR (composite of Limbach and Microsynth laboratory results) detected $236(42.2 \%)$ samples as Plasmodium infections; of these, 173 (73.3\%) were identified as $P$. vivax, 57 (24.2\%) as $P$. falciparum, and six $(2.5 \%)$ as mixed-species infections. Neither Plasmodium ovale nor Plasmodium malariae infections were identified. By microscopy, a total of $182(32.6 \%)$ samples were identified as Plasmodium infections; of these, $138(75.8 \%)$ were identified as $P$. vivax, 42 (23.8\%) as $P$. falciparum, and two (1.1\%) as mixed-species infections.

A good agreement to detect Plasmodium infections was shown between the Loopamp Malaria Pan Detection Kit and the combined PCR techniques (Cohen's Kappa 0.8266, 95\% Cl: 0.7792-0.874). Using the combined PCR as the reference method, the Loopamp Malaria Pan Detection Kit showed a sensitivity of $87.7 \%$ (95\% Cl: $83.5-91.9)$ and a specificity of 94.4\% (95\% Cl: 91.9-96.9). At the same time, microscopy showed a lower sensitivity of $77.1 \%$ (95\% Cl: 71.8-82.5) and a higher specificity of $100 \%$ (Table 1 ).

For detection of $P$. vivax infections, the Loopamp Malaria Pv Detection Kit showed a sensitivity of $84.4 \%(95 \% \mathrm{Cl}$ : 79.0-89.7) and a specificity of $92.4 \%$ (95\% Cl: 89.7-95.0), with a substantial agreement with PCR (Cohen's kappa: $0.7661,95 \%$ Cl: 0.7088-0.8234). (Table 2), whereas the microscopy had a sensitivity of $77.7 \%(95 \% \mathrm{Cl}: 71.6-83.8)$ and a specificity of $99.7 \%$ (95\% Cl: $99.2-100)$, with Cohen's kappa of 0.8212 (95\% Cl: 0.7693-0.8731). Considering a scenario where only Pan LAMP-positive samples would be tested with the Loopamp Malaria Pv Detection Kit, the positive predictive value and negative predictive value would have been 83.9 (78.5-89.3) and 92.6 (90-95.2), respectively.

The Loopamp Malaria Pv Detection Kit identified 29 (5.1\%) positive samples that were not detected as $P$. vivax by PCR techniques (Figure 1). One of these was a sample classified as a mixed infection by microscopy and as a $P$. falciparum infection by PCR; three were identified as $P$. falciparum only by PCR; 10 were identified as $P$ falciparum by microscopy and PCR, suggesting a failure in the specificity of the primer set; and the remaining 15 apparently false-positive sample were detected as Plasmodium by the Loopamp Malaria Pan Detection Kit. This finding of apparent false positives was reported in previous studies of the Loopamp Malaria Pan Detection Kit and could be explained by discrepancies related to the stochastic processes expected in detection of low parasitemia infections. ${ }^{13,17-19}$

The low density may have influenced the diagnostic accuracy; for seven of the 15 apparently false-positive samples, interobserver discrepancy was noted when reading the results of both LAMP assays. The interobserver differences in the Loopamp Malaria Pv Detection Kit was 9.1\% (51/559),

TABLE 2

Performance evaluation of microscopy and the Loopamp Malaria Pv Detection Kit for Plasmodium vivax identification, using PCR as the reference method

\begin{tabular}{|c|c|c|c|c|c|}
\hline \multirow{3}{*}{$\begin{array}{l}\text { Plasmodium vivax } \\
\text { infections }\end{array}$} & Statistic & \multicolumn{2}{|c|}{ Microscopy } & \multicolumn{2}{|c|}{ Pv loop-mediated isothermal amplification } \\
\hline & True negative & 379 & & 351 & \\
\hline & True positive & 139 & & 151 & \\
\hline & False positive & 1 & & 29 & \\
\hline & False negative & 40 & & 28 & \\
\hline & Sensitivity (95\% Cl) & 77.7 & (71.6-83.8) & 84.4 & $(79.0-89.7)$ \\
\hline & Specificity (95\% Cl) & 99.7 & $(99.2-100)$ & 92.4 & (89.7-95.0) \\
\hline & Positive predictive value (95\% Cl) & 99.3 & $(99.7-100)$ & 83.9 & (78.5-89.3) \\
\hline & negative predictive value $(95 \% \mathrm{Cl})$ & 90.5 & (87.6-93.3) & 92.6 & $(90.0-95.2)$ \\
\hline & Cohen's kappa & 0.8212 SE 0.0265 & $(0.7693-0.8731)$ & 0.7661 SE 0.0292 & $(0.7088-0.8234)$ \\
\hline
\end{tabular}




\section{A Plasmodium detection}

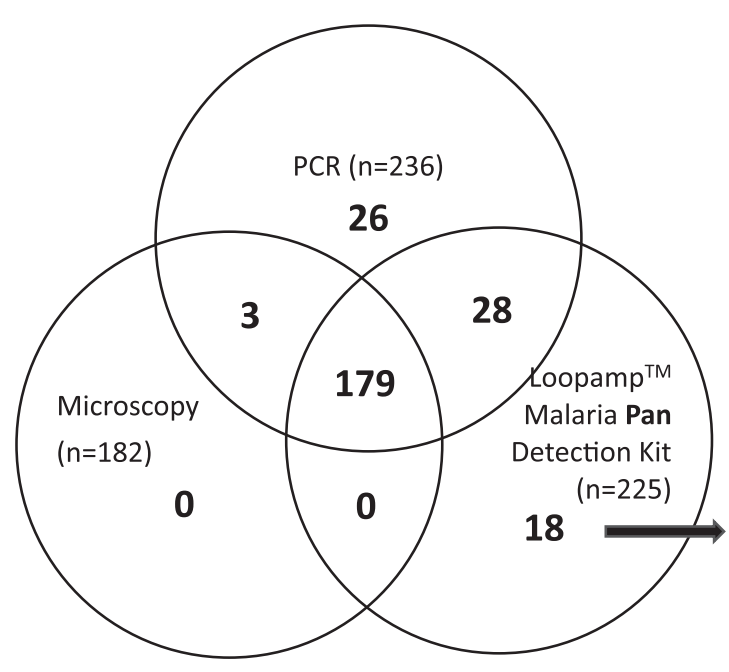

\section{B Plasmodium vivax detection}

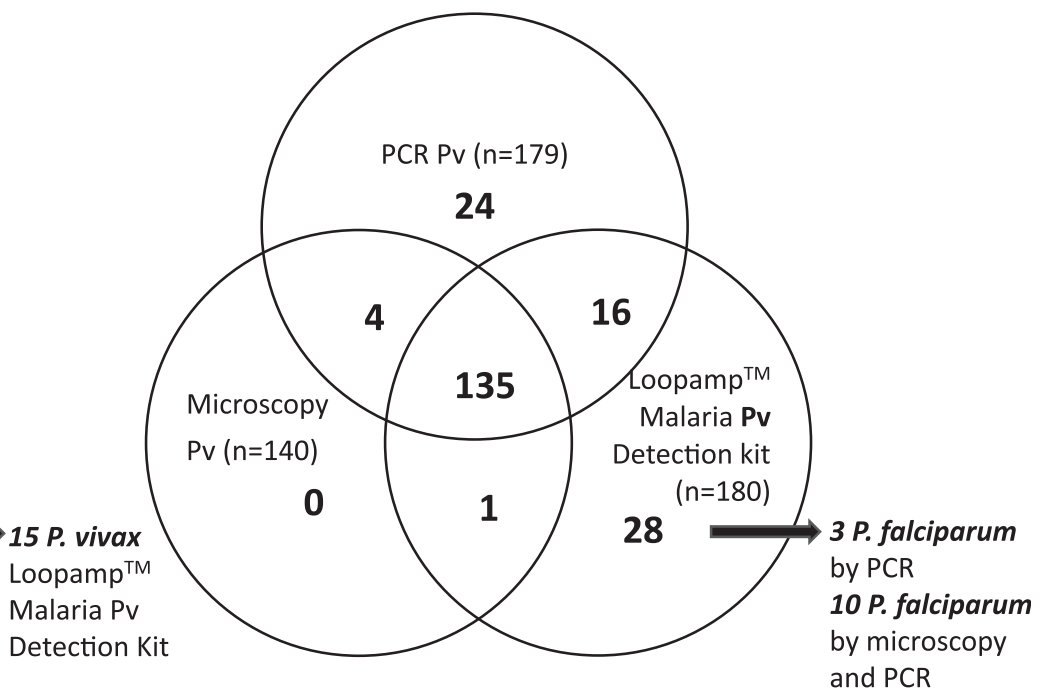

15 Plasmodium Loopamp ${ }^{\mathrm{TM}}$

Malaria Pan

Detection kit

Figure 1. Plasmodium (A) Plasmodium vivax (B) infection identification according to three diagnostic methods.

whereas for the Loopamp Malaria Pan Detection Kit was 7.5\% (42/559).

In conclusion, the performance of a new commercial Loopamp Malaria Pv Detection Kit was consistent with that of an already available Loopamp Malaria Pan Detection Kit, with a superior sensitivity than microscopy performed in this study by two independent readers and highly skilled personnel. This suggests that the LAMP diagnosis could be very useful in rural and remote areas of Peru, where malaria incidence is high, and microscopic diagnosis does not always meet the quality requirements owing to limited resources. However, more studies would be needed to evaluate the operational feasibility and cost-effectiveness of implementing LAMP in such settings than the costs and operational difficulties of maintaining highquality microscopy diagnosis.

The definite identification of $P$. vivax, which is needed to provide adequate radical treatment, could be effectively performed by combining the use of a Loopamp Malaria Pan Detection Kit and Loopamp Malaria Pv Detection Kit. However, because of the rate of false identification of $P$. falciparum as $P$. vivax seen in this study $(7.2 \%[13 / 180])$, the combined use with the Loopamp ${ }^{\mathrm{TM}}$ Malaria Pan/Pf Detection Kit would be a much better option for making adequate treatment decisions.

This report focuses on the use of LAMP with febrile individuals attending a health facility, but the performance of this new assay should also be evaluated in asymptomatic low parasitemia infections, where the superior sensitivity of LAMP would likely be more critical. There might be various clinical scenarios where LAMP will likely be a useful tool in malaria elimination programs; however, important aspects for its implementation, such as training of health workers and management of shelf life and storage conditions of the reagents in POC settings, should be evaluated in remote communities with limited resources.
Received March 24, 2020. Accepted for publication May 18, 2020

Published online August 3, 2020.

Note: Supplemental file appears at www.ajtmh.org.

Acknowledgments: We thank laboratory technician Yovana Maldonado and the staff of the Malaria Laboratory of Universidad Peruana Cayetano Heredia, who were involved in the processing of clinical samples.

Financial support: This work was financially supported by FIND (Geneva, Switzerland) using grants awarded by the Australian government and by the Paul G. Allen Family Foundation. O. N. is supported by the project "Translational Research Development for Endemic Infectious Diseases of Amazonia"- FOGARTY. Project number: 2D43TW007120-11A1.

Authors' addresses: Oscar Nolasco and Juan Contreras-Mancilla, Instituto de Medicina Tropical "Alexander von Humboldt" Universidad Peruana Cayetano Heredia, Lima, Perú, E-mails: oscar.nolasco.c@ upch.pe and juan.contreras.m@upch.pe. Beronica Infante, Laboratorios de Investigación y Desarrollo, Facultad de Ciencias y Filosofía, Universidad Peruana Cayetano Heredia, Lima, Perú, E-mail: beronica.infante.g@upch.pe. Sandra Incardona and Xavier C. Ding, FIND, Geneva, Switzerland, E-mails: sandra.incardona@finddx.org and xavier.ding@finddx.org. Dionicia Gamboa and Katherine Torres, Instituto de Medicina Tropical "Alexander von Humboldt" Universidad Peruana Cayetano Heredia, Lima, Perú, Laboratorios de Investigación y Desarrollo, Facultad de Ciencias y Filosofía, Universidad Peruana Cayetano Heredia, Lima, Perú, and Departamento de Ciencias Celulares y Moleculares, Facultad de Ciencias y Filosofía, Universidad Peruana Cayetano Heredia, Lima, Perú, E-mails: dionigamboa@ yahoo.com and katherine.torres.f@upch.pe.

This is an open-access article distributed under the terms of the Creative Commons Attribution (CC-BY) License, which permits unrestricted use, distribution, and reproduction in any medium, provided the original author and source are credited.

\section{REFERENCES}

1. Dirección General de epidemiología. Ministerio de Salud, 2018. Sala Situacional Para el Análisis de Situación de Salud-Semana 
52. Lima, Peru. Available at: https://www.dge.gob.pe/portal/ docs/vigilancia/sala/2018/salaSE52.zip. Accessed March 20, 2020.

2. Rosas-Aguirre $A$ et al., 2015. Hotspots of malaria transmission in the Peruvian Amazon: rapid assessment through a parasitological and serological survey. PLoS One 10: e0137458.

3. Rovira-Vallbona E, Contreras-Mancilla JJ, Ramirez R, GuzmánGuzmán M, Carrasco-Escobar G, Llanos-Cuentas A, Vinetz JM, Gamboa D, Rosanas-Urgell A, 2017. Predominance of asymptomatic and sub-microscopic infections characterizes the Plasmodium gametocyte reservoir in the Peruvian Amazon. PLoS Negl Trop Dis 11: e0005674.

4. Ministerio de Salud, Instituto Nacional de Salud, 2015. Norma Técnica de Salud Para la Atención de la Malaria y Malaria Grave en el Peru. Available at: https://cdn.www.gob.pe/uploads/ document/file/322855/Norma_t\%C3\%A9cnica_de_salud_ para_la_atenci\%C3\%B3n_de_la_malaria_y_malaria_grave en_el_Per\%C3\%BA20190621-17253-idp4k7.pdf. Accessed March 20, 2020.

5. Kilian AHD, Metzger WG, Mutschelknauss EJ, Kabagambe G, Langi P, Korte R, Sonnenburg F, 2000. Reliability of malaria microscopy in epidemiological studies: results of quality control. Trop Med Int Health 5: 3-8.

6. Okell LC, Ghani AC, Lyons E, Drakeley CJ, 2009. Submicroscopic infection in Plasmodium falciparum-endemic populations: a systematic review and meta-analysis. J Infect Dis 200: 1509-1517.

7. Berzosa $\mathrm{P}$ et al., 2018. Comparison of three diagnostic methods (microscopy, RDT, and PCR) for the detection of malaria parasites in representative samples from Equatorial Guinea. Malar J 17: 333.

8. Endeshaw T et al., 2008. Evaluation of light microscopy and rapid diagnostic test for the detection of malaria under operational field conditions: a household survey in Ethiopia. Malar J 7: 118.

9. Jang JW, Cho CH, Han ET, An SS, Lim CS, 2013. pLDH level of clinically isolated Plasmodium vivax and detection limit of $\mathrm{pLDH}$ based malaria rapid diagnostic test. Malar J 12: 181.
10. Imwong M, Hanchana S, Malleret B, Rénia L, Day NP, Dondorp A, Nosten F, Snounou G, White NJ, 2014. High-throughput ultrasensitive molecular techniques for quantifying low-density malaria parasitemias. J Clin Microbiol 52: 3303-3309.

11. Britton S, Cheng Q, McCarthy JS, 2016. Novel molecular diagnostic tools for malaria elimination: a review of options from the point of view of high-throughput and applicability in resource limited settings. Malar J 15: 88.

12. Notomi T, Okayama $\mathrm{H}$, Masubuchi $\mathrm{H}$, Yonekawa $\mathrm{T}$, Watanabe $\mathrm{K}$, Amino N, Hase T, 2000. Loop-mediated isothermal amplification of DNA. Nucleic Acids Res 28: E63.

13. Serra-Casas E et al., 2017. Loop-mediated isothermal DNA amplification for asymptomatic malaria detection in challenging field settings: technical performance and pilot implementation in the Peruvian Amazon. PLoS One 12: e0185742.

14. Haanshuus C, Mohn S, Mørch K, Langeland N, Blomberg B, Hanevik K, 2013. A novel, single-amplification PCR targeting mitochondrial genome highly sensitive and specific in diagnosing malaria among returned travellers in Bergen, Norway. Malar J 12: 12-26.

15. Perkins S, Schall J, 2002. A molecular phylogeny of malarial parasites recovered from cytochrome $b$ gene sequences. J Parasitol 88: 972-978.

16. Foundation for Innovate New Diagnostic, 2012. Manual of standard operating procedures for malaria LAMP. Available at: https://www.finddx.org/wp-content/uploads/2016/04/SOPLAMP-Malaria-Aug2012.pdf. Accessed March 20, 2020.

17. Hopkins $\mathrm{H}$ et al., 2013. Highly sensitive detection of malaria parasitemia in a malaria-endemic setting: performance of a new loop-mediated isothermal amplification kit in a remote clinic in Uganda. J Infect Dis 208: 645-652.

18. Cook $\mathrm{J}$ et al., 2015. Loop-mediated isothermal amplification (LAMP) for point-of-care detection of asymptomatic lowdensity malaria parasite carriers in Zanzibar. Malar J 14: 43.

19. Polley SD, 2013. Clinical evaluation of a loop-mediated amplification kit for diagnosis of imported malaria. $J$ Infect Dis 208: 637-644. 